\title{
Study on Trade-off between Orientation Compression and Fingerprint Recognition Performance
}

\author{
Xuying Zhao \\ Beijing Electronic Science and Technology Institute \\ NO.7 Fufeng Road, Fengtai District, Beijing, 100070, \\ P.R.China \\ xyzhao@besti.edu.cn
}

\begin{abstract}
Integrating mutual information of fingerprint orientation with minutia template matching result can enhance the performance of fingerprint recognition system. To reduce the size of fingerprint template data in embedded system, an improved run length encoding algorithm for fingerprint template compression is proposed. The trade-offs between orientation compression and fingerprint recognition performance is studied. Several experimental tests have been conducted and results show the good performance of the algorithm.
\end{abstract}

Keywords-Run-length encoding, Fingerprint orientation field, Pattern recognition

\section{INTRODUCTION}

Most of the fingerprint verification systems required to provide a high processing speed and high degree of security are prominently based on minutiae matching and several efficient minutia-based matching algorithms have been proposed [1-3]. Fingerprint minutia template consists of minutia type, coordinates, ridge direction, curvature and iner-ridge number, etc., that need less than 10 Bytes of storage. There are on average 50 valid minutiae in which the principle categories are ridge endings and bifurcations that represent the local fine characteristics.

Combination of minutiae information that is essential local features of fingerprint and orientation information describing the global patterns can improve the performance of the fingerprint recognition system. In this paper, mutual information is proposed to evaluate similarity of two fingerprints that is descried as a powerful measure for the registration of multimodality medical images which have showed great promise and have been used in a wide variety of applications [4-8]. Grey values of image pixels are subtituted by fingerprint orientation fields to estimate mutual information because of its high noise immunity that has been used for fingerprint registration [9].

Several approaches to estimation of fingerprint orientation fields have been represented that is computed in blocksize [10-13]. Jian's method [10] is adopted in this paper in which the fingerprint image is divided into blocks of a fixed size of $w \times w$ pixels. Howerever, fingerprint orientation fields need large storage capability that is not suited for embedded system. Generally, $w=8$, it requires $37 \times 37=1369$ Bytes of storage, as an example, for a fingerprint image of size $300 \times 300$ pixels.

\author{
Xiaokun Zhang, Geng Zhao, Kejun zhang, Rong Qian, \\ Yan Zhang \\ Beijing Electronic Science and Technology Institute \\ \{sam, zg, zkj, rqian, zhangyan\}@besti.edu.cn
}

\begin{abstract}
Therefore, it is necessary to study trade-offs between fingerprint orientation storage and recognition performance of embedded system. Run Length Encoding (RLE) is a popular data compression algorithm that is supported by most bitmap file formats, such as TIFF, BMP, and PCX [14-15]. This paper proposes an impoved RLE method of fingerprint orientation fields that are firstly quantized in an appropriate interval.

This paper is arranged as follows. Section 2 provides the algorithm for estimation of fingerprint orientation fields, while approaches to quantization and compression of fingerprint orientation fields are described in section 3 . We present fingerprint matching method combining orientation and minutia information in section 4. In section 5, experimental results and conclusions are given finally.
\end{abstract}

\section{ESTIMATION OF FINGERPRINT ORIENTATION FIELDS} Gradients $G_{x}(x, y)$ and $G_{y}(x, y)$ at each pixel $(x, y)$ of an image are defined by

$$
\left[\begin{array}{c}
G_{x}(x, y) \\
G_{y}(x, y)
\end{array}\right]=\left[\begin{array}{c}
\frac{\partial I(x, y)}{\partial x} \\
\frac{\partial I(x, y)}{\partial y}
\end{array}\right],
$$

where $I(x, y)$ denotes grey value of pixel $(x, y)$.

Direction angle $\alpha$ at $(x, y)$ can be given by

$\alpha=\frac{1}{2} \operatorname{artan}\left(\frac{2 G_{x} G_{y}}{G_{x}^{2}-G_{y}^{2}}\right)$.

Fingerprint orientation fields represent global texture information that vary slowly at adjacent pixles, so the fingerprint image is divided into blocks of a fixed size of $w \times w$ pixels, and direction angle $\theta(i, j)$ of each block centered in $(i, j)$ is computed by 


$$
\theta(i, j)=\frac{1}{2} \operatorname{artan}\left(\frac{\sum_{u \in-\frac{w}{2}} \sum_{v=j-\frac{w}{2}}^{i+\frac{w}{2}} 2 G_{x}(u, v) G_{y}(u, v)}{\sum_{u+i-\frac{w}{2}}^{j+\frac{w}{2}} \sum_{v=j-\frac{w}{2}}^{j+\frac{w}{2}}\left[G_{x}^{2}(u, v)-G_{y}^{2}(u, v)\right]}\right) .
$$

Finally, some smoothing work is carried out on the orientation fields and in general, fingerprint orientation angle is limited to $\left[-\frac{\pi}{2}, \frac{\pi}{2}\right)$ by

$$
\phi(i, j)=\left\{\begin{array}{ll}
\theta(i, j)+\pi / 2 & \theta(i, j) \leq 0 \\
\theta(i, j)-\pi / 2 & \theta(i, j)>0
\end{array} .\right.
$$

\section{QUANTIZATION AND COMPRESSION}

RLE is a lossless algorithm that offers decent compression ratios in specific types of data and is a good introduction into the data compression field including video, voice, images, text, music, etc.. The algorithm is based on the idea to replace a long sequence of the same symbol by a shorter sequence. As far as a fingerprint image is concerned, orientation data have approximate characteristic of ascending or descending order in a local area. Consequently, the deviation between two directional angles of successive blocks is calculated and only the first block orientation value is stored while others are substituted with frequence of identical deviation.

Suppose the quantization parameter is $\mathrm{N}$, then the orientation angle of the No.1 block can be stored in the first $\mathrm{N}$ bits of a Byte, while the other $8-\mathrm{N}$ bits contain the frequence of the same deviation. Accordingly, the quantization interval is defined by equally dividing $\left[-\frac{\pi}{2}, \frac{\pi}{2}\right)$ by $2^{N}$ and the direction angle is assigned to an integer value by equation:

$$
I \phi(i, j)=\left[\frac{\phi(i, j)}{\pi} \times 2^{N}\right]+2^{N-1},
$$

where [] means the nearest integer function. Accordingly, Fingerprint orientation template consists of block-size orientation fields that the angle value is quantized in the range from 0 to $2^{\mathrm{N}}-1$. Simulation results in embedded sysytem with 16 bit processor are shown in Figure. 1 when $\mathrm{N}=5$.

As shown in Figure.1 (a), RLE is implemented along the positive $\mathrm{Z}$ direction. The quantized orientation values in the labelled area and the results of compression storage have been shown in Figure.1 (b), while an example of 16 bit storage is shown in Figure.1 (c).

\section{FINGERPRINT MATCHING}

A rigid transformation is determined to register two fingerprint images using minutiae coordinates and minutiae angles of corresponding pairs. The query fingerprint is lapped over to the reference one and the fingerprint orientation fields are recalculated on the transformed image with the same blocksize, as shown in Figure.2, where two images in (a) are captured from the same finger and those images in (b) are from different fingers.

The joint probability distribution $P_{X Y}(x, y)$ of the orientation fields can be calculated by counting the number of times $p_{n}(x, y)$ each quantized orientation value occurs in the overlapping area and dividing those numbers by the total number of occurrences, that is

$$
P_{X Y}(x, y)=\frac{p_{n}(x, y)}{\sum_{x=0}^{2^{N}-1} \sum_{y=0}^{2^{N}-1} p_{n}(x, y)} .
$$

The marginal probability distribution, $p_{X}(x)$ and $p_{Y}(y)$, of query and reference fingerprint can be obtained by

$$
\begin{aligned}
& P_{X}(x)=\sum_{y=0}^{2^{N}-1} P_{X Y}(x, y), \\
& P_{Y}(y)=\sum_{x=0}^{2^{N}-1} P_{X Y}(x, y) .
\end{aligned}
$$

The normalized mutual information of two fingerprint images that is less sensitive to changes in overlap can be given by

$$
M I=\frac{\sum_{x} p_{X}(x) \log p_{X}(x)+\sum_{y} p_{Y}(y) \log p_{Y}(y)}{\sum_{x, y} p_{X Y}(x, y) \log p_{X Y}(x, y)}
$$

MI is a measure of dependence between two fingerprints considering that there is maximal dependence between the orientation fields if they are from the same finger and correctly registered, while unrelated fingerprints or misalignment will result in a decrease in the value. To evaluate the similarity between two fingerprints, the matching score is calculated as a measure of the similarity level integrating with minutia features and orientation information using the product fusion strategy. 


\section{EXPERIMENTAL RESULTS AND CONCLUSIONS}

The experiments reported in this paper have been conducted on Pattek fingerprint database in CASIA that the images were captured by the capacitor sensor of Veridicom company. The database contains 4000 fingerprint images of size $300 \times 300$ pixels captured at a resolution of $500 \mathrm{dpi}$, from 200 fingers with 20 impressions per finger, which vary in quality, direction and position.

A set of experiments are implemented to test the compression ratio and the performance of the fingerprint recongnition system using the same minutiae sets, as shown in Table.1. From the results we can see that the method proposed in this paper outperforms the algorithm based on minutia matching and a fairly high average compression ratio is achieved while the best parameter $\mathrm{N}=4$.

In conclusion, this paper studies trade-offs between compression ratio and recognition performance and the optimization is obtained. Additionally, combining MI of orientation and minutia matching result can improve the performance of the fingerprint recognition system

\section{REFERENCES}

[1] A.K. Jain; S. Prabhakar; L. Hong; S. Pankanti. Filterbank-based fingerprint matching, IEEE Trans. Image Processing, 9(5), 2000, 846-859.

[2] F. Gamble; L. Frye; D. Grieser. Real-Time fingerprint verification system, Applied Optics, 31(5), 1992, 652-655.

[3] C.J. Lee; S.D. Wang. Fingerprint feature extraction using gabor filters, Electronics Letters, 35(4), 1999, 288-290.

[4] Pluim J.P.W.; Maintz J.B.A.; Viergever M.A.. Mutual-InformationBased Registration of Medical Images A Survey. IEEE Transactions on Medical Imaging, Volume 22, Issue 8, Aug. 2003 Page(s):986 1004.

[5] R. P. Woods; S. R. Cherry; J. C. Mazziotta; Rapid automated algorithm for aligning and reslicing PET images. journal of computer assisted tomography, vol. 16, no. 4, pp. 620-633, 1992.

[6] A. Collignon; F. Maes; D. Delaere; D. Vandermeulen; P. Suetens; G. Marchal; Automated multi-modality image registration based on information theory. Information Processing in Medical Imaging, 1995, pp. 263-274.

[7] Studholme; D. L. G. Hill; D. J. Hawkes. An overlap invariant entropy measure of 3D medical image alignment. Pattern Recognition, vol. 32, no. 1, pp. 71-86, 1999.

[8] F. Maes; D. Vandermeulen; P. Suetens. Comparative evaluation of multiresolution optimization strategies for multimodality image registration by maximization of mutual information. Medical Image Analysis, vol. 3, no. 4, pp. 373-386, 1999.

[9] Lifeng Liu; Tianzi Jiang; Jianwei Yang; Chaozhe Zhu. Fingerprint registration by maximization of mutual information. IEEE Transactions on Image Processing, Volume 15, Issue 5, May 2006 Page(s): 1100 - 1110.

[10] Kalle Karu; Anil Jian. Fingerprint Classfication, Pattern Recognition (1996), Vol.29, No.3

[11] M. Kass; A. Witkin. Analyzing Oriented Patterns. Computer Vis. Graphics image Processing, 37, 1984, pp.263-285

[12] Jie Zhou; Jinwei Gu. A model-based method for the computation of fingerprints' orientation field. IEEE Transactions on Image Processing, Volume 13, Issue 6, June 2004, P.821 - 835

[13] Dongdg Nie; Lizhuang Ma; XueZhong Xiao; Shuangjiu Xiao. Optimization Based Fingerprint Direction Field Estimation. Proceedings of 27th Annual International Conference of the Engineering in Medicine and Biology Society 2005 (IEEE-EMBS 2005). Page(s):6265 - 6268

[14] www.data-compression.info/Algorithms/RLE/index.htm

[15] Khalid Sayood. Introduction to Data Compression, Third Edition, Morgan Kaufmann, Dec. 1, 2005

TABLE I. CONTRAST OF COMPRESSION AND MATCHING PERFORMANCE

\begin{tabular}{|c|c|c|c|}
\hline Compression & \multicolumn{2}{|c|}{ Equal Error Rate (EER) } & Average Compression \\
\cline { 2 - 2 } Parameter & Our Method & Minutia Matching & Ratio (16 bit) \\
\hline $\mathrm{N}=3$ & 0.396 & \multirow{3}{*}{0.485} & 0.196 \\
\hline $\mathrm{N}=4$ & 0.251 & & 0.213 \\
\hline $\mathrm{N}=5$ & 0.265 & & 0.363 \\
\hline
\end{tabular}




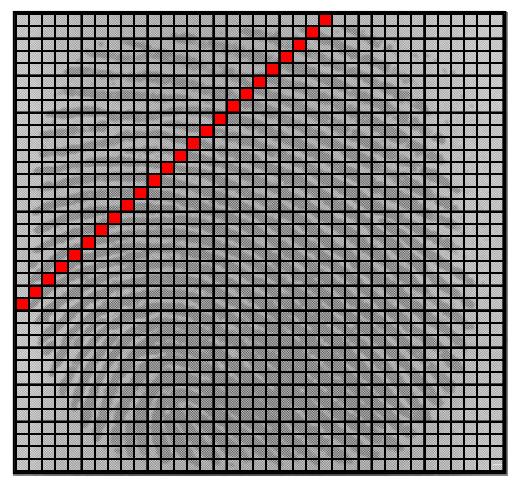

(a)

\begin{tabular}{|c|c|c|c|c|c|c|c|c|}
\hline Block Coordinates & $(24,1)$ & $(23,2)$ & $(22,3)$ & $(21,4)$ & $(20,5)$ & $(19,6)$ & $(18,7)$ & $(17,8)$ \\
\hline Quantized Value & 8 & 8 & 4 & 4 & 4 & 4 & 4 & 5 \\
\hline Compression and Storage & $0 \times 08 \mid 0 \times 00$ & $0 \times 08 \mid 0 \times 00$ & $0 \times 04 \mid 0 \times 00$ & $0 \times 04 \mid 0 \times 03$ & & & $0 \times 05 \mid 0 \times 00$ \\
\hline Coordinates & $(16,9)$ & $(15,10)$ & $(14,11)$ & $(13,12)$ & $(12,13)$ & $(11,14)$ & $(10,15)$ & $(9,16)$ \\
\hline Quantized Value & 5 & 6 & 7 & 8 & 8 & 9 & 9 & 9 \\
\hline Compression and Storage & $0 \times 05 \mid 0 \times 00$ & $0 \times 06 \mid 0 \times 02$ & \multicolumn{7}{|c|}{$0 \times 08 \mid 0 \times 00$} & $0 \times 09 \mid 0 \times 00$ & $0 \times 09 \mid 0 \times 09$ \\
\hline Coordinates & $(8,17)$ & $(7,18)$ & $(6,19)$ & $(5,20)$ & $(4,21)$ & $(3,22)$ & $(2,23)$ & $(1,24)$ \\
\hline Quantized Value & 9 & 9 & 9 & 9 & 9 & 9 & 9 & 9 \\
\hline Compression and Storage & 9 & 9 & \multicolumn{7}{|c|}{} \\
\hline
\end{tabular}

(b)

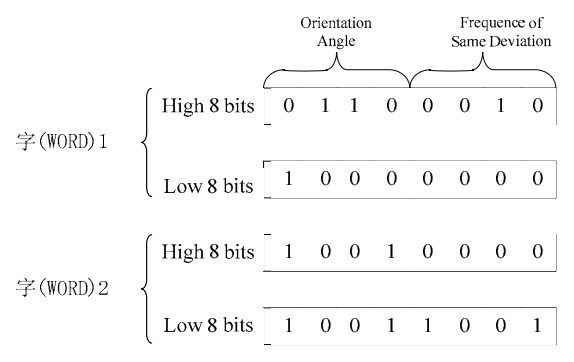

(c)

Figure 1. Instantiation of orientation quantization and compression in 16 bit system

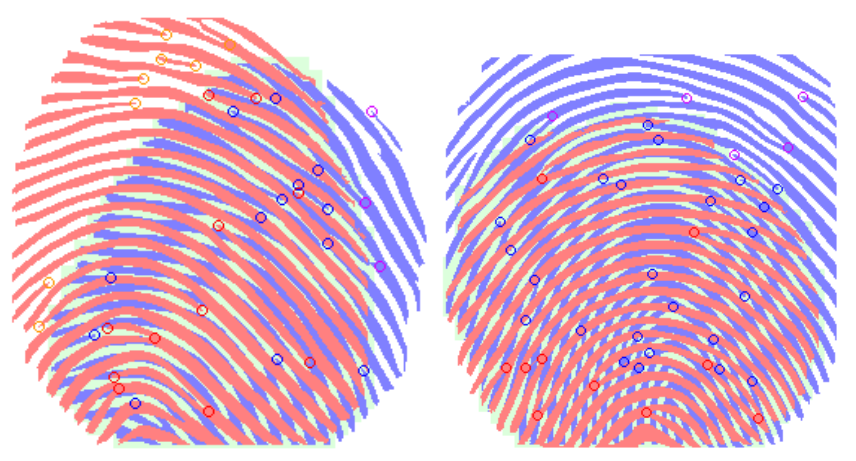

(a)

(b)

Figure 2. Alignment of two fingerprint images 